\title{
NASADEM GLOBAL ELEVATION MODEL: METHODS AND PROGRESS
}

R. Crippen ${ }^{*}$, S. Buckley, P. Agram, E. Belz, E. Gurrola, S. Hensley, M. Kobrick, M. Lavalle, J. Martin, M. Neumann, Q. Nguyen, P. Rosen, J. Shimada, M. Simard, W. Tung

Jet Propulsion Laboratory, California Institute of Technology, Pasadena, California 91109 USA

Robert.E.Crippen@jpl.nasa.gov

Commission IV, WG IV/3

KEY WORDS: Elevation, Topography, DEM, NASADEM, SRTM, ASTER, GDEM, ICESat

\begin{abstract}
:
NASADEM is a near-global elevation model that is being produced primarily by completely reprocessing the Shuttle Radar Topography Mission (SRTM) radar data and then merging it with refined ASTER GDEM elevations. The new and improved SRTM elevations in NASADEM result from better vertical control of each SRTM data swath via reference to ICESat elevations and from SRTM void reductions using advanced interferometric unwrapping algorithms. Remnant voids will be filled primarily by GDEM3, but with reduction of GDEM glitches (mostly related to clouds) and therefore with only minor need for secondary sources of fill.
\end{abstract}

\section{INTRODUCTION}

NASADEM will be NASA's successor digital elevation model (DEM) to the currently available "SRTM Plus" (NASA SRTM Version 3), which consists of SRTM Version 2 (the watermasked original SRTM) with voids filled primarily by ASTER GDEM2 or secondarily by GMTED2010 or the (US) National Elevation Dataset (NASA JPL, 2013). NASADEM moves forward by first taking a step back to reprocess the original SRTM radar data, now using software and reference ancillary data (from ICESat) that did not exist for the original processing, which occurred soon after the Shuttle Radar Topography Mission in 2000.

Filling voids that remain in the new SRTM DEM and extension of NASADEM to higher latitudes focuses on the soon-to-beavailable GDEM3. GDEM3 is the latest (and expected last) near-global DEM derived from near-infrared stereoscopic measurements provided by the ASTER imaging sensor on the Terra satellite. We have developed methods for post-processing improvement of GDEM3 that greatly increase its utility in NASADEM.

\section{METHODS}

\subsection{Improved Interferometric Unwrapping of SRTM}

SRTM used the radar interferometric technique to map the topography of most of Earth's land surface (Farr et al., 2007). The method was successful in producing accurate elevation measurements for about $99 \%$ of the imaged area within the latitude range N60 to S56. The remaining problem areas consisted mostly of very rugged terrain, which (in simple terms) commonly had radar layover or shadows, and very smooth terrain, where little of the side-looking radar signal was reflected back to the sensor.

Advances in synthetic aperture radar (SAR) interferometry since the SRTM mission include the SPAPHU method of Chen and Zebker (2002), which involves using interferometric tiles that are assembled into a maximally probable full-size solution. A benefit of this approach is that, with some caution, the topography of additional terrains can be determined that might otherwise fail to unwrap.

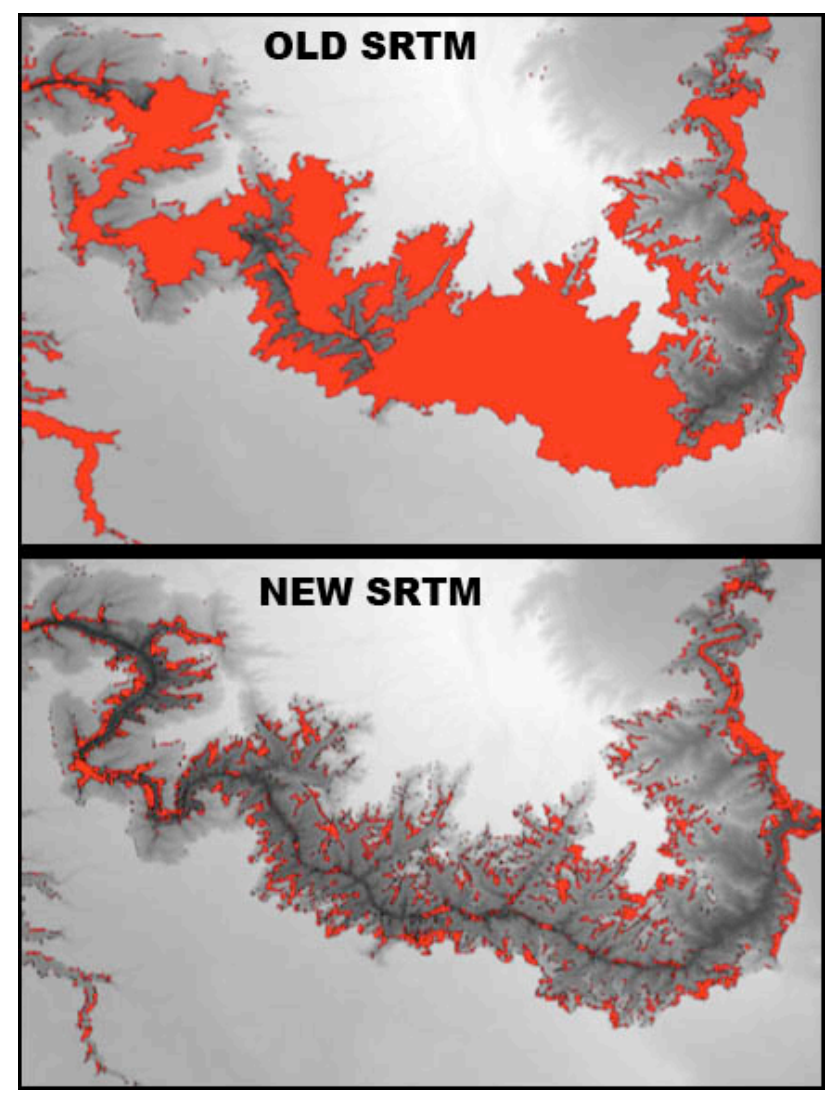

Figure 1. Voids in red, original versus reprocessed SRTM. Height as brightness, Grand Canyon, Arizona, USA.

\footnotetext{
${ }^{*}$ Corresponding author
} 
Figure 1 shows an example of the use of the new unwrapping strategy to reduce voids in the reprocessed SRTM DEM compared to the original SRTM DEM. The area shows the Grand Canyon in Arizona, USA, a distinctive and extremely steep and rugged terrain. Much of the main canyon area was void (shown as red) in the original DEM (top) but is now filled with new SRTM elevation values (bottom). Much smaller voids remain, but SRTM control of secondary DEM fill (such as GDEM or even interpolation) improves exponentially with decreasing void size.

\subsection{ICESat Precision Adjustment of SRTM}

Interferometric unwrapping calculates relative measures of surface elevation across a terrain. Affixing the DEM to absolute heights requires reference to independently known elevations at some locations. The original SRTM project

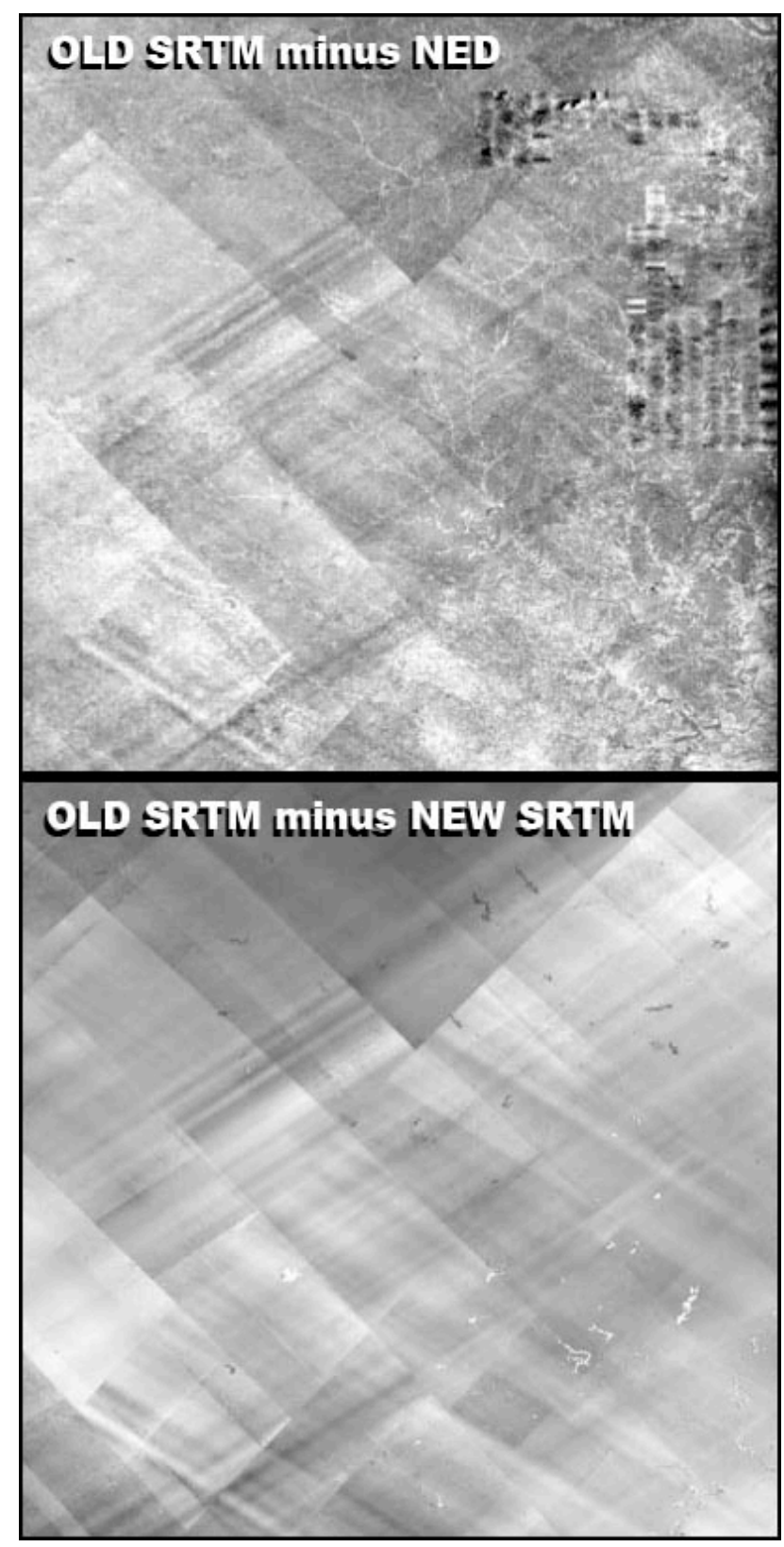

Figure 2. Old SRTM swath height errors evident versus both NED and the new SRTM, in which they have been reduced. Great Plains, USA, N35-40 W95-100. accomplished that by using a global scatter of ground control points (GCPs) and also water levels at ocean coasts (with tide models), which were effective because of the continental scale of the data takes. This worked well, but lack of GCP density limited the absolute vertical accuracy to several (5-10) meters (Rodriguez et al., 2006). Also, crossing swaths (from ascending orbits versus descending orbits) and adjacent swaths (from nearby orbits) used differing (and far and near) GCPs, resulting in steps between swaths in the final DEM mosaic.

In order to improve the vertical accuracy and consistency of the swaths, and uniformity within the swath mosaic, NASADEM applies vertical and tilt adjustments based upon GCPs derived from the ICESat laser altimeter. ICESat (Schutz et al., 2005) was launched in 2003 and operated until 2009. Our studies indicate that land cover heights measured by SRTM generally correspond to the 50\% energy return level (RH50) of ICESat (meaning that as a forest canopy opens, SRTM elevations get closer to the bare ground while the midpoint of the total energy return signal of ICESat is also closer to the ground). This use of ICESat has been effective, and we have recently added temporal filters to the ICESat data to suppress glacial melting and deforestation temporal differences between SRTM and ICESat.

In Figure 2 we demonstrate the success of the method by examining a $5 \times 5$ degree area generally covering the Great Plains region of Kansas and Oklahoma, USA. This low-relief terrain is targeted in order to minimize errors inherent in complex topography. In the top panel we difference the original SRTM DEM from the National Elevation Dataset (NED) in order to show the swath pattern problem. Actual topographic features drop out of the difference while SRTM and NED errors remain. The NED errors generally have north-south and eastwest alignments (especially evident in the upper right of the top panel), while SRTM swaths are aligned NW-SE and NE-SW and commonly differ by six meters in height. The bottom panel differences the original SRTM DEM from the new SRTM DEM that uses ICESat GCPs. Note that the SRTM swath pattern removed (bottom) closely matches the original SRTM error pattern (top). Remnant swath difference errors, evident in the difference of new SRTM minus NED (not shown), are mostly no greater than one meter.

\subsection{GDEM3 Refinement: Cloud Removal}

The series of ASTER Global Elevation Models (GDEM) used stereo correlation in each of millions of ASTER scenes in order to measure topographic height as a function of parallax. GDEM2 (Fujisada et al., 2012) provided spatial resolution and general quality improvements over GDEM1. GDEM3, the expected final version, is now available to us and is under evaluation by us in coordination with the U.S. Geological Survey and the U.S. and Japanese ASTER Science Teams. GDEM3 uses hundreds of thousands of additional ASTER scenes for the goal of quality improvement.

As with previous GDEM versions, topographic spikes (and less commonly pits) occur in GDEM3 in some locations. These errors are usually spatially small but can be extremely tall because they are usually related to cloud elevations that are imperfectly eliminated from the optical stereo elevation calculations. We have developed a method to eliminate most spikes (and deep pits) directly from GDEM with no ancillary data. In essence, our method detects, eliminates, and replaces topographic features in GDEM that are not realistic natural topography. The method is complex but starts with a measure of curvature (rather than slope), and then applies spatial filters. 
Figure 3 shows an example of GDEM spike removal in a cloudy tropical area (S01E010, Gabon, Africa). In most cases spike removal is virtually complete with no adverse effects to the surrounding real terrain. We have also been evaluating the extent to which this method can be used outside the SRTM latitude range to improved GDEM in polar areas.
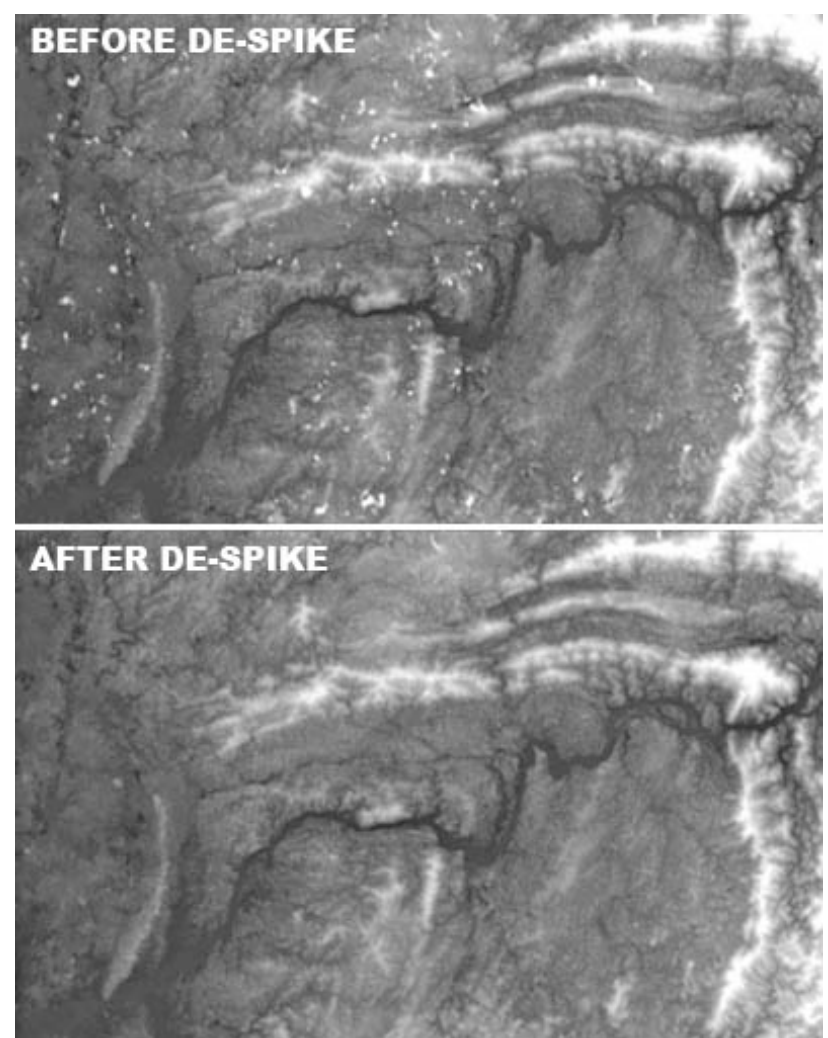

Figure 3. Cloud spike removal from GDEM3 in Gabon, height as brightness.

\subsection{Fixing the SRTM Water Mask}

SRTM Version 1 is called the "unfinished" SRTM DEM. It included all elevation values produced by interferometric unwrapping, including noisy and incomplete values on water bodies. This is the same type of output that our new SRTM DEM will have. SRTM Version 2, the much more widely useful version of SRTM, employed a water mask to flatten, calibrate the height for, and spatially complete the water bodies (Slater et al., 2006). We will use the same water mask, but we have found several readily noticeable errors in the mask.

Fortunately, GDEM3 was produced with a new and additional product: a water mask derived from ASTER imagery. Although the ASTER water mask has some serious errors of its own, it appears to very well correct most of the errors in the SRTM water mask (e.g. Figure 4). Thus, selective application of the ASTER water mask to the SRTM water mask will improve the "finishing" step for our new SRTM DEM.

\subsection{SRTM-GDEM Merger Improvements}

SRTM Plus (NASA SRTM Version 3) used a modified version of the Delta Surface Fill method (Grohman et al., 2006) to seamlessly merge GDEMv2 with SRTMv2. NASADEM will use the same (or very similar) method. But it is worth noting that the worst results in SRTM Plus were attributable to clouds in GDEM. This was often where clouds were within the GDEM fill areas, but it was also (even worse) where the GDEM clouds were at the SRTM void edges (the merger seams). GDEM was rejected where it mismatched SRTM by 80 meters or more at the seams. At and near those locations, the much lower resolution (comparatively blurry) GTOPO2010 DEM was usually used instead of GDEM. The trouble with this was that a small cloud in GDEM at the edge of a large void in SRTM would exclude GDEM from much of the void fill, wasting all of the good GDEM elevations in that area.

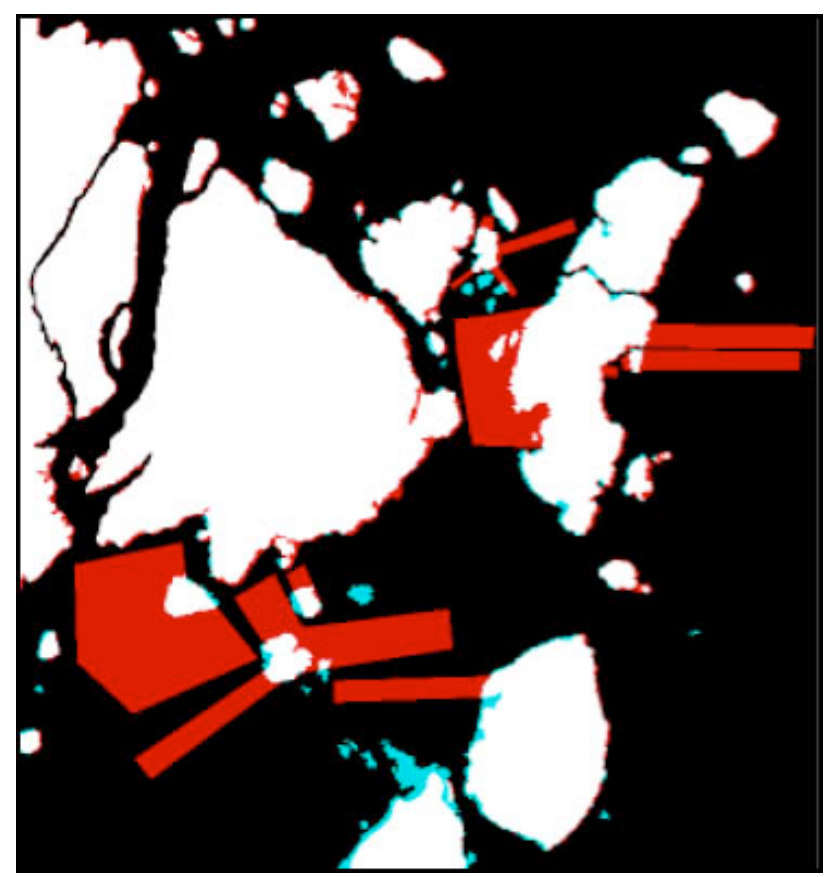

Figure 4. SRTM Water Body Database errors corrected to water (red) and to land (cyan) via the GDEM water mask. Sulu Archipelago, Philippines, N05E120. Black $=$ water.

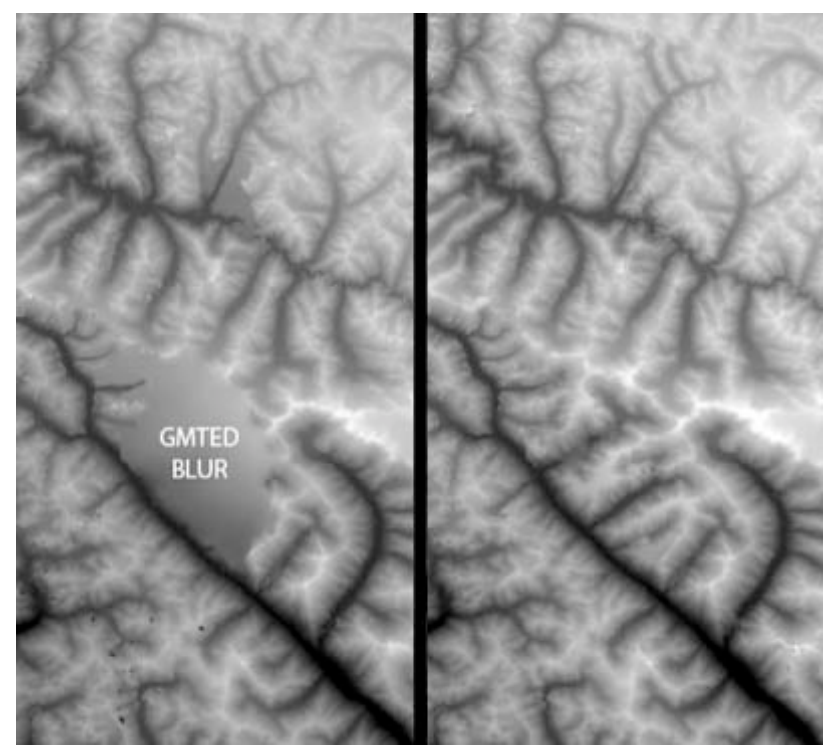

Figure 5. Southernmost central China, N29E096. Left: SRTM

Plus with large blur area (GMTED2010) due to GDEM2 rejection related to cloud spikes. Right: NASADEM inclusion of GDEM3 after cloud spike removal. 
With cloud avoidance now expected (discussed above), we have successfully tested a new arrangement for the DEM mergers. GDEM will now be de-spiked and then filled with an alternate DEM, or interpolated) and then used to seamlessly fill SRTM. This has been shown to indeed greatly increase the availability of good GDEM elevation pixels in areas that previously ended up as blur (or error) from GMTED2010. An example is shown in Figure 5.

\section{PRODUCTS AND COMPLETION}

The main product from the NASADEM project is, obviously, the DEM. Completion is expected in late 2017. Some products, including the new pre-merger, pre-water-masked SRTM-only DEM, will be made available as floating point data, which has some added value particularly for hydrologic studies. Additional products will include the pixel-by-pixel error estimates and geoid-relative slope, aspect, and curvature measures, calculated with weighted consideration of those error estimates. The interferometric correlations will also be made newly available, which have some value, for example, in vegetation mapping. It should be kept in mind that the SRTM mission was a unique, 10-day radar "snapshot" record of the status of Earth's surface in the year 2000.

\section{ACKNOWLEDGEMENTS}

This work was performed at the Jet Propulsion Laboratory, California Institute of Technology, under a contract with the National Aeronautics and Space Administration.

\section{REFERENCES}

Chen, C., and H. Zebker, 2002. Phase unwrapping for large SAR interferograms: Statistical segmentation and generalized network models. IEEE Transactions on Geoscience and Remote Sensing, 40(8), pp. 1709-1719.

Farr, T., P. Rosen, E. Caro, R. Crippen, R. Duren, S. Hensley, M. Kobrick, M. Paller, E. Rodriguez, L. Roth, D. Seal, S. Shaffer, J. Shimada, J. Umland, M. Werner, M. Oskin, D. Burbank, and D. Alsdorf, 2007. The Shuttle Radar Topography Mission. Reviews of Geophysics, volume 45, doi:10.1029/2005RG000183.

Fujisada, H., M. Urai, and A. Iwasaki, 2012. Technical methodology for ASTER global DEM. IEEE Transactions on Geoscience and Remote Sensing, 50(10), pp. 3725-3736.

Grohman, G., G. Kroenung, and J. Strebeck, 2006. Filling SRTM voids: The delta surface fill method. Photogrammetric Engineering and Remote Sensing, 72(3), pp. 213-216.

NASA JPL, 2013. NASA Shuttle Radar Topography Mission Global 1 arc second DEM, Version 3. NASA LP DAAC. http://doi.org/10.5067/MEaSUREs/SRTM/SRTMGL1.003

Rodriguez, E., C. Morris, and J. Belz, 2006. A global assessment of the SRTM performance. Photogrammetric Engineering and Remote Sensing, 72(3), pp. 249-260.

Schutz, B., H. Zwally, C. Shuman, D. Hancock, and J. DiMarzio, 2005. Overview of the ICESat Mission. Geophysical Research Letters, volume 32, doi:10.1029/2005GL024009.
Slater, J., G. Garvey, C. Johnston, J. Haase, B. Heady, G. Kroenung, and J. Little, 2006, The SRTM data "finishing" process and products. Photogrammetric Engineering and Remote Sensing, 72(3), pp. 237-247. 